\title{
Les crues de juillet 1997 en République Tchèque
}

\author{
July 1997 floods in the Czech Republic
}

\author{
par H. Molicova \\ Générale des Eaux, Vivendi
}

\section{J. Hladny}

Responsable du service hydrologique opérationnel auprès de l'Institut Hydro-météorologique de la République Tchèque.

In July 1997, in East Europe long lasting intense rainfalls took place. Floods in Moravia, East Bohemia brought about fifty deaths, 10 billion dollars losses and 29000 habitations and hundreds of industrial plants devastation. In order to analyse all aspects of rainfall-flow processes (from meteorologic causes in the troposphere to changes in the underground water after the occurence), a pluridisciplinary team was set up. Their conclusions may lead to measures for preparing the coming back of such extreme phenomena.

\section{I LE RISQUE PERMANENT DES CRUES.}

Les catastrophes naturelles représentent sans doute un événement extraordinaire dans une vie humaine. Par contre la nature n'attribue à ces phénomènes extrêmes aucun caractère exceptionnel. Les écologues les qualifient de perturbations [1] et soulignent même leur importance pour les écosystèmes. Ainsi de ce point de vue, la notion de dégâts n'aurait pas lieu d'exister. Ce sont seulement les humains qui subissent les dommages, et cela par des forces naturelles qui échappent à leur contrôle. Cela est valable pour les inondations que l'on devrait considérer comme une partie du cycle de l'eau.

Selon l'Organisation Météorologique Mondiale la surface terrestre reçoit quotidiennement en moyenne des centaines de milliers $\mathrm{m}^{3}$ d'eau sous forme de précipitations. A cause de la répartition hétérogène de ces dernières il existe aussi bien des régions dont le climat est très sec que des régions bénéficiant d'apports pluviométriques intenses. On peut donc assister quotidiennement dans différentes parties de la Terre à une vingtaine de drames où l'eau quitte le lit des cours d'eau et devient un élément dangereux pour l'Homme et ses biens. Les nuisances liées aux débordements des cours d'eau dans le monde entier ont été répertoriés pour la dernière décennie. Ces faits impliquent quelques leçons dont il faut tenir compte dans l'hydrologie moderne pour mener à bien la protection contre les inondations :

- Le cycle de l'eau est permanent. Nécessairement, il fait partie des phénomènes assurant le fonctionnement du système planétaire. Ainsi, dans des conditions où l'eau est évacuée d'une façon concentrée, le risque d'occurrence d'une crue demeure permanent. C'est pour cela que la protection contre les crues devrait être menée en permanence et elle devrait considérer les particularités des conditions physicogéographiques.

- Le cycle de l'eau se déroule dans divers environnements et il est déterminé par une combinaison de différents processus. Si l'on doit alors éclaircir le mécanisme de genèse d'une crue et de son déroulement il faut impérativement inclure tous les liens nécessaires à la prise en compte de la complexité des milieux et des chaînes de causalité. C'est pour cette raison que l'étude d'une crue ne constitue pas seulement un problème du point de vue de la gestion de l'eau, de la météorologie et de l'hydrologie, mais qu'elle doit être entreprise dans le cadre d'une problématique interdisciplinaire incluant l'hydraulique, l'écologie, la géographie, la géologie, la pédologie, la morphologie, la chimie, l'hygiène etc.

- Dans la nature, les crues extrêmes apparaissent de façon très irrégulière dans le temps comme dans l'espace. C'est pour cela que chaque événement de crue peut apporter un enseignement sur la façon de se protéger et de limiter les effets indésirables provoqués des crues. Pour se préparer efficacement à affronter des crues on peut également étudier les crues historiques.

- L'Homme ne devrait pas perdre sa vigilance lors des périodes calmes entre deux crues dévastatrices, périodes parfois assez longues à l'échelle de la vie humaine, comme dans le cas de la succession de crues nombreuses mais modestes. A ce propos la figure 1 compare le nombre d'occurrences saisonnières et l'importance des débits maximaux des crues dans le bassin versant de la rivière Labe (Elbe) à Decin (République Tchèque) au cours de périodes d'observation de 50 ans. On peut ainsi constater que les 
crues ont été beaucoup plus nombreuses et certainement plus conséquentes au cours du $\mathrm{XIX}^{\mathrm{e}}$ siècle.

\section{L'ANALYSE DES CAUSES MÉTÉORO- LOGIQUES}

Les crues catastrophiques du mois de juillet 1997 font partie des manifestations extrêmes du cycle de l'eau qui peuvent aléatoirement affecter l'Europe avec des puissances et des capacités destructrices inégales dans l'espace. Cette fois-ci le fléau a frappé sur des parties du territoire de l'Autriche, de la République Tchèque, de la Pologne, de l'Allemagne et de la Slovaquie [2].

La cause initiale ayant provoqué les crues en Europe Centrale a été l'existence d'un front froid en provenance du sudouest. Son passage a été ralenti dans la région alpine, ce qui a permis l'arrivée par la vallée du Rhône de masses d'air froid vers la Méditerrannée. Ainsi s'est créée une zone dépressionnaire qui, lors de son passage vers le nord-est, a donné lieu à des précipitations abondantes (figure 2), en particulier dans la partie orientale de la Bohême et dans la partie méridionale de la Pologne. Ensuite, un anticyclone en provenance des Açores se déplaçant vers la Scandinavie du sud a bloqué la dépression au-dessus de la Pologne du sud. Cette cause d'ordre météorologique a donc contribué à une très longue période de précipitations intenses sur le territoire de la République Tchèque qui ont provoqué la première série de crues du 4 au 8 juillet 1997.

Le gradient entre les hautes et les basses pressions s'aggravant alors que la vitesse des vents du Nord augmentait. C'est pour ces raisons que l'effet de versant a pu jouer considérablement sur les parties Nord et Nord-Est de la Bohême septentrionale.

La genèse de la seconde vague de précipitations fut différente, même si vers le 17 juillet les deux fronts, l'un se déplaçant à travers l'Europe centrale l'autre vers l'est à travers la Méditerranée, se sont rejoints. Plus tard et jusqu'au 21 juillet, le développement synoptique fut analogue à celui du premier système dépressionnaire. Les apports pluviométriques de la seconde vague de précipitations furent cependant inférieurs à ceux de la première, n'atteignant que 30 à $50 \%$ des apports de celle-ci. En comparant les causes météorologiques des deux épisodes de crues, on constate une coïncidence apparente issue des caractéristiques connues de l'atmosphère : les processus de circulation atmosphérique ont une tendance à se reproduire. Du point de vue hydrosynoptique le stationnement au-dessus de la Pologne méridionale de la dépression responsable des crues constitue un événement singulier car il s'agit d'une situation qui n'a jamais été observée depuis le début de ce siècle. Pour la République Tchèque cette période a été à la fois la plus longue période pluvieuse et celle où les précipitations ont été les plus intenses.

\section{III — LES PRÉCIPITATIONS EXTRÊMES}

La figure 3 montre les trajectoires suivies par les dépressions ayant provoqué des crues historiques sur le bassin versant de la rivière Odra à Bohumín. Il a été observé qu'il s'agissait toujours de cyclones venant du sud selon la trajec-

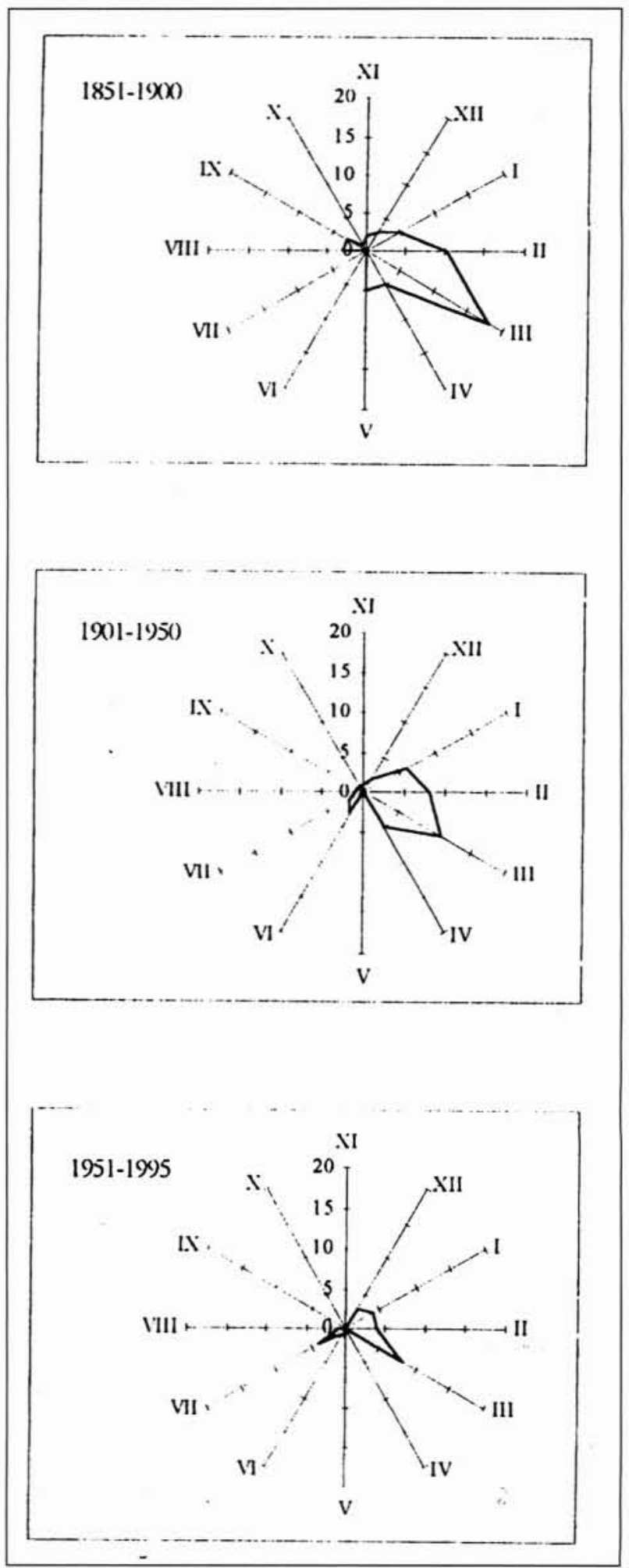

1. Les fréquences (à gauche) et les débits (à droite) saisonniers de la rivière Labe (Elbe) à Decin.

toire $V_{b}$, du Nord de I'Italie au Sud de la Pologne. Il faut donc chercher les causes des crues extrêmes de juillet 1997 sur la rivière Odra dans la coexistence et l'interaction des facteurs hydrosynoptiques, de la trajectoire des cyclones, de leur structure verticale, de leur taux d'humidité, des effets de versant et d'un éventuel effet "entonnoir" résultant de la configuration du relief [3]. 


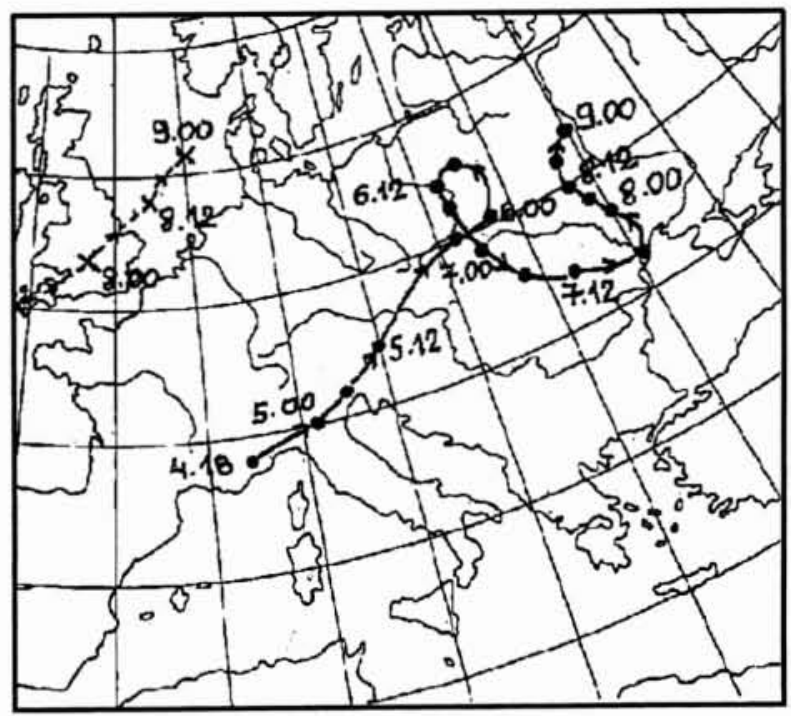

2. Les trajectoires des épicentres des anticyclones (+) et des cyclones $\left({ }^{\circ}\right)$ du 4 au 9 juillet 1997

On a montré que les champs de nombreuses variables météorologiques ont présenté des valeurs proches des extrêmes pendant la période de crues de juillet 1997 et c'est la combinaison de ces variables qui a permis aux précipitations d'atteindre un niveau extrême. Il en découle qu'une estimation quantitative de la probabilité d'un tel événement doit s'appuyer sur une analyse croisée des probabilités des facteurs explicatifs des précipitations.

Une fine prévision météorologique des champs pluviométriques dans le temps et dans l'espace constitue une problématique majeure pour permettre au service de la protection contre les crues de faire appel aux modèles prévisionnels des crues utilisant comme donnée de base des totaux pluviométriques. Les connaissances citées plus haut exigent, néanmoins, une prise en compte dans ces modèles d'autres arguments dans le cadre du "postprocessing ", basé sur une estimation significative des probabilités multidimensionnelles de l'élément inconnu [4].

L'extension géographique des précipitations intenses durant plusieurs jours, avec une période de retour de 500 à 1000 ans en Moravie septentrionale peut être considérée sans doute comme un événement exceptionnel. Cependant, si l'on examine les apports pluviométriques journaliers et leurs totaux sur deux jours, ce phénomène ne paraît plus aussi extrême.

\section{IV — RÉPONSE DES BASSINS VERSANTS}

Les deux séries de précipitations intenses en juillet 1997 ont provoqué deux vagues de crue. Au cours de la première série pluviométrique ayant eu lieu du 4 au 8 juillet, les inondations se manifestaient violemment en amont des cours d'eau déjà le 7 juillet au matin et elles ont atteint les parties médianes des cours d'eau le 8 ou le 9 juillet. Même si la seconde série pluviométrique, du 17 au 21 juillet a été moins abondante en apports pluviométriques, l'amont de la rivière Morava a encore connu des inondations intenses car les effets des deux vagues se sont conjuguées. En ce qui concerne la deuxième crue les débits ont atteint leur paroxysme pour la

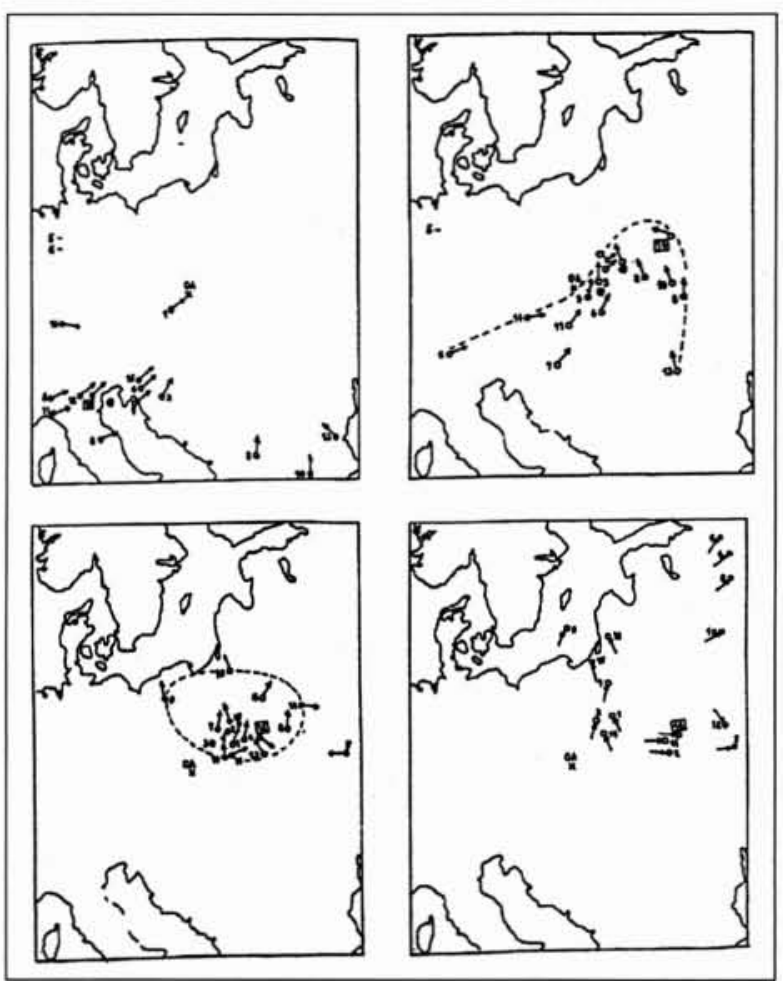

3. Les épicentres des cyclones responsables des crues sur la rivière Odra à Bohumin pour la période 1896-1997 (D $=$ jour du paroxysme de la crue, $o=$ centre du cyclone, $\Rightarrow$ sa direction, $O A=$ localisation de la ville Ostrava (République Tchèque), 16 = le cas du cyclone responsable pour la première vague des crues).

plupart des cours d'eau en amont et en partie médiane le 19 juillet et en aval de la rivière Morava le 22 juillet 1997.

Les vagues de crue en amont des régions montagneuses ont été brusques - pour la rivière Odra et en amont de la rivière Morava, la cote du plan d'eau de la rivière a monté de 2 à 6 mètres. La vitesse de l'écoulement s'est également accélérée pour atteindre des valeurs extrêmes, dépassant même $5 \mathrm{~m} \cdot \mathrm{s}^{-1}$, ce qui a provoqué des effets dévastateurs sur le lit de cours d'eau et sur les aménagements environnants [5].

La durée des précipitations intenses a eu pour conséquence la conjonction néfaste des vagues de crues venant des différents cours d'eau et la concentration des masses d'eau en aval (localement le coefficient d'écoulement a dépassé la valeur 0,8$)$. Les coefficients d'écoulement spécifique dans les zones les plus touchées par la pluie ont approché la valeur de $3 \mathrm{~m}^{3} \cdot \mathrm{s}^{-1} \cdot \mathrm{km}^{-2}$ [6].

En aval et dans les parties médianes de la rivière Morava et dans les dépressions des autres cours d'eau, I'extension des inondations a atteint localement même plusieurs kilomètres. Ce débordement a retardé la progression de la vague de crue et l'a immobilisée dans les zones affectées. Les inondations ont ainsi couvert la surface de $1248 \mathrm{~km}^{2}$, soit $5 \%$ de la superficie des bassins versants concernés.

\section{V — L'ÉVOLUTION DE L'ÉCOULEMENT}

A la lumière des mesures débitmétriques effectuées au cours des crues de juillet 1997, les débits caractéristiques pour les différentes périodes de retour ont été réexaminés. Cette analyse montre que globalement les périodes de retour 
des débits culminants pour les crues de juillet 1997 diminuent vers l'aval. Par exemple, en ce qui concerne la rivière Morava, en amont, dans la localité Raskov, la période de retour du débit maximal a été 800 ans, vers l'aval à Olomouc cette dernière n'a atteint que 500 ans, à Kromeriz 300 ans et à Straznice seulement 100 ans. Dans le bassin versant de la rivière Odra, cette tendance a été moins prononcée. En Bohême de l'Est et dans les bassins versants des rivières Moravska Sazava, Trebuvka, Svitava et Svratka et Becva les périodes de retour des débits maximaux n'ont même pas dépassé 200 ans.

Les débits centennaux $\left(Q_{100}\right)$ estimés à partir des données comprenant les débits culminants des crues de juillet 1997 ont été comparés aux données équivalentes antérieures. Cette confrontation suggère pour certains bassins versants l'élaboration de nouvelles abaques des débits caractéristiques pour toute une gamme de périodes de retour [7].

Ensuite les périodes de retour des hauteurs pluviométriques ayant causé les crues de juillet 1997 ont été comparées à celles des débits culminants et des élévations de la cote du plan d'eau des rivières. Il apparait que les périodes de retour de la cote du plan d'eau des rivières ont été plus courtes que celles correspondant aux hauteurs pluviométriques.

\section{VI $\square$ INFLUENCES DES AMÉNAGEMENTS SUR LE TAUX D'INONDATIONS}

La transformation des débits de crue a été assurée à l'aide de 24 bassins de rétention à capacité totale de 124,2 millions de $\mathrm{m}^{3}$, soit $3,4 \%$ de l'équivalent du volume des précipitations de la période du 4 au 8 juillet 1997. Ces bassins influençaient l'écoulement sur une surface d'environ $5000 \mathrm{~km}^{2}$ [8].

Il est apparu que pour certains ouvrages les capacités de rétention d'eau n'ont pas été pleinement utilisées : selon les normes en vigueur la réserve d'eau initiale n'a pas été évacuée au début de la crue et les premières arrivées d'eau, dont la propagation n'aurait pas affecté l'aval des barrages, ont déjà été retenues - ces mesures ont ainsi limité la capacité et l'effet de rétention au moment stratégique.

Les rôles des retenues d'eau plus modestes ont été évalués séparement pour 316 bassins avec une superficie totale estimée pour les cotes habituelles des plans d'eau à 3800 ha. Ces dispositifs offrent des possibilités d'absorber des crues avec une période de retour de l'ordre de 20 à 30 ans. Cependant, pour les crues de juillet 1997, leurs effets n'ont été utiles qu'à une échelle très locale. Ils étaient souvent remplis au maximum et quelques dizaines des barrages ont été débordés et parfois même renversés, sans que cela ait forcément des conséquences tragiques.

\section{VII — L'INFUENCE DU MODE D'OCCUPA- TION ET DE L'ÉTAT DU SOL SUR LA RÉTENTION DE LA CRUE}

Les travaux de modélisation ont suggéré que le mode d'occupation du sol par les cultures et le mode de leur exploitation et la répartition des forêts et des prairies influencent la capacité d'infiltration du sol. Ainsi ils constituent des facteurs agissant directement sur la répartition de l'écoule- ment de crue en composantes de surface et de subsurface lors de la phase initiale de chaque crue.

On ne peut pas prétendre que les sols affectés par la série des crues de juillet 1997 ont déjà été saturés, donc imperméables, dès le début des crues suite à des apports pluviométriques antérieurs abondants. Au contraire, le processus d'infiltration a été dès le début de la crue très efficace et ainsi il a réduit l'écoulement superficiel. Après la saturation complète du sol l'infiltration a baissé en fonction de l'écoulement subsuperficiel. La rétention du sol a donc pu jouer dans la phase d'augmentation des débits alors que pendant le paroxysme des crues son effet a été moindre [9].

La levée cartographique des sols alluviaux retranche l'histoire du paysage et les cartes correspondantes peuvent être utilisées comme indicateur de l'espace inondable. Ainsi, la comparaison des sols alluviaux avec les limites d'inondations liées au paroxysme des crues de juillet 1997 a montré qu'il s'agissait des événements majeurs qui se sont, néanmoins, déjà produits à plusieurs reprises.

Quant au drainage organisé, les simulations ont montré que ce dernier a pu compter pour $2-5 \%$ des eaux présentes dans les espaces drainés au moment du paroxysme des crues de juillet 1997. Il a été estimé qu'une lame de 15 à $25 \mathrm{~mm}$ pouvait s'infiltrer en plus dans les terrains drainés par rapport à ceux n'ayant subi aucun traitement.

Les plus grands dommages du réseau hydrographique, et au-delà les transformations du paysage ainsi que les ravages des villages entiers ont été causés surtout par l'érosion dans les régions montagneuses (Jeseniky, Beskydy) et par une sédimentation massive des matériaux érodés.

Les terrains alluviaux, surtout en amont des bassins versants, ont souvent subi des pertes et la dévalorisation du sol productif. L'aval et les parties médianes des cours d'eau ont été exposés à des sédimentations massives et à un appauvrissement (év. concentration) des substances chimiques, certaines localités ayant subi des inondations prolongées ont même enregistré des processus de réduction.

Ces conséquences dramatiques ont mis en exergue l'urgence de la mise en cuvre des mesures de protection contre l'érosion et la nécessité d'un aménagement du territoire orienté vers une stabilisation du paysage. Cependant, les capacités de rétention du sol pouvant être influencées par le mode d'occupation et d'exploitation du sol, même utilisées au maximum n'auraient pas pu absorber les quantités d'eau mobilisées par un événement tel que la série des crues de juillet 1997 en République Tchèque.

\section{VIII — QUALITÉ DES EAUX DE SURFACE}

Les variations de la qualité des eaux de surface ont été observées déjà lors de la première vague des crues de juillet 1997. Les concentrations ont surtout augmenté pour les substances insolubles, les matières organiques, les métaux et la conductivité, même si ces concentrations n'ont pas dépassé leurs valeurs maximales pour la période 1992-1996. Par contre, une baisse de concentration, à des teneurs minimales enregistrées pendant la même période, a été constatée pour les chlorures, le calcium, l'azote ammoniacal, le phosphore total et, à l'exception du bassin versant de Morava, pour l'azote des nitrates [10]. 
La crue a provoqué le transfert énorme de flux de matières, dépassant les valeurs maximales observées pour la période de 1992-1996, pour tous les indicateurs suivis à l'exception de l'azote ammoniacal. Les plus grands flux ont été observés pour les mêmes indicateurs dont les concentrations étaient les plus hautes (les substances insolubles, les matières organiques et les métaux).

Les concentrations de la matière en suspension ont été proches des valeurs moyennes pour la période 1986-1995. Les concentrations maximales ont été atteintes lors de la période d'accroissement rapide des débits de la première vague de crue. Quant à la deuxième série des crues, les concentrations maximales n'ont même pas atteint la moitié de celles de la première crue. Par contre, les flux de matières en suspension ont atteint des niveaux extrêmes, multipliés plusieurs fois par rapport aux moyennes estimées à long terme.

Les analyses de la teneur des métaux lourds dans la matière en suspension ont constaté leur baisse par rapport à la moyenne de la période 1986-1995 sauf pour le nickel, le fer et dans une moindre mesure pour le mercure. Leurs flux mesurés en matière en suspension ont néanmoins atteint des valeurs importantes.

Les plaines inondées ont connu un accroissement de l'alcalinité, du calcium et du phosphore total, ainsi que de la concentration de certains métaux lourds, qui n'ont néanmoins pas dégradé la qualité des eaux, au moins du point de vue de la norme en vigueur. Cependant, la qualité des eaux s'est fortement dégradée par la pollution (PCB, AOX, PAU) venant des sites industriel inondés surtout lors de la première vague des crues. Les observations de l'évolution de ces concentrations peuvent être utiles pour l'optimisation des travaux d'inondation et de drainage.

Les pannes des stations d'épuration des eaux usées n'ont été ressenties, au niveau des indicateurs tels que la DCO, l'azote ammoniacal, le phosphore total et le contenu bactérien, qu'à la fin du mois de juillet 1997. Ces crises n'ont pas seulement affecté des cours d'eau mais elles ont touché aussi la qualité des eaux souterraines. Au total les régions affectées ont subi plus que 60 fuites de substances dangereuses pour l'environnement telles que les dérivés pétroliers et les huiles minérales.

\section{IX — EAUX SOUTERRAINES}

Le milieu hydrogéologique a montré une grande capacité d'absorbtion des eaux, ce qui a contribué à modérer les conséquences des crues. Des simulations mathématiques ont estimé une infiltration de 60 à $150 \mathrm{~mm}$ selon les sites. La piézométrie de la plupart des aquiferes n'a retrouvé son niveau habituel qu'au mois de janvier, voire février 1998 [11].

La qualité des eaux souterraines a été analysée sur les sites choisis exploitant l'eau des formations quaternaires des rivières Morava et Odra. Ces études ont mis en évidence la pollution par des bactéries et l'accroissement des teneurs en sulfates et localement des hydrocarbures. Par contre, la teneur en chlorure et dans une moindre mesure en nitrates a baissé.

Les captages d'eau potable étant contaminés, l'alimentation en eau potable ne pouvait pas être correctement assurée.
Cette crise a mis en évidence l'extrême urgence de prévoir des solutions de secours et l'importance de protéger des ressources stratégiques.

\section{DOCUMENTATION GÉODÉSIQUE}

Les résultats des travaux géodésiques au sol et de la télédétection ont été examinés en détail, en particulier pour certains segments des cours d'eau des rivières Morava, Becva et Odra. Ces informations ont ensuite été vérifiées sur le terrain. L'ensemble de cette documentation toujours en cours de mise à jour permet de dégager l'enveloppe délimitant les régions sinistrées. En vue de projets d'aménagement du territoire, il a été élaboré un modèle numérique de terrain pour les segments de la rivière Odra et Morava [12].

\section{XI — CONCLUSIONS}

L'objectif de ce travail n'a pas été tellement de présenter les résultats du projet consacré à l'étude d'un événement aussi singulier mais de souligner l'emprise de ce projet d'envergure mené dans un cadre pluridisciplinaire. Il est clair que les mesures de prévention peuvent passer seulement par la connaissance des causes et des mécanismes responsables du déclenchement des phénomènes aussi extrêmes. Les moyens investis dans cette étude peuvent être valorisés si on arrive à tirer des leçons de ces acquis venant des domaines divers.

\section{BIBLIOGRAPHIE}

[WMO] : Disaster Around the World - A Global and Regional View. Information Paper for World Conference on Natural Disaster Reduction, Geneva Switzerland, May 1994.

[1] Sтовсн, D. : O katastrofach malych a velkych. Vesmir 77, Praha, octobre 1998.

[2] Strachota, J. et al.: Meteorologické príciny povodnové situace. Praha, CHMÚ, cerven 1998

[3] KaKos, V. : Posouzeni vyjimecnosti hydrosynopticke situace na zaklade srovnani s dostupnymi historickymi pripady. In : Meteorologicke priciny povodnove situace v cervenci 1997, Praha, CHMU, juin 1998.

[4] Sokol. Z. et Rezacova D. : Lokalni predpoved srazek pomoci statistickeho zpracovani vystupu numerickych predpovednich modelu - statisticky postprocessing. Meteorologicke Zpravy 51, 4, 1998.

[5] HaVLIK, A. et al.: Hydraulické vyhodnocení dynamiky odtoku a rozlivu. Praha, Vúv T.G.M., juin 1998.

[6] SERCL, P. et al.: Hodnocení prubehu povodnovych vin. Praha, CHMÚ, juin 1998.

[7] Kulasova, B. et al.: Hodnocení extremity kulminacních prutoku a objemu povodnovych vln. Praha, CHMÚ, juin 1998.

[8] MaCHACEK, L. et al.: Vliv nádr í a dalsích vodohospodárskych del na povodnovou situaci. Praha, VD-TBD, juin 1998.

[9] JANECEK, M. et al.: Vliv stavu a vyu ívání krajiny na povod novou situaci. Praha-Zbraslav, VÚMOP, juin 1998.

[10] Rosendorf, P. et al.: Vliv povodne na jakost povrchovych a podzemních vod. Praha, VúV T.G.M., juin 1998.

[11] Kesst., J. et al.: Vliv povodnové situace na obeh podzemních vod. Praha, CHMÚ, juin 1998.

[12] Kolar, V. et al:: Geodetická dokumentace pro vyhodnocení povodne a tvorbu digitálního modelu rícního systému. Praha, VUV T.G.M., cerven 1998. 